\title{
Proteomic insights into the lifestyle of an environmentally relevant marine bacterium
}

\author{
Joseph Alexander Christie-Oleza ${ }^{1}$, Bernard Fernandez ${ }^{1}$, Balbina Nogales ${ }^{2}$, Rafael Bosch ${ }^{2}$ \\ and Jean Armengaud ${ }^{1}$ \\ ${ }^{1}$ Laboratoire de Biochimie des Systèmes Perturbès, CEA Marcoule, DSV, IBEB, Bagnols-sur-Cèze, France \\ and ${ }^{2}$ Departament de Biologia, Microbiologia, Universitat de les Illes Balears, Palma de Mallorca, Spain
}

\begin{abstract}
In terms of lifestyle, free-living bacteria are classified as either oligotrophic/specialist or opportunist/ generalist. Heterogeneous marine environments such as coastal waters favour the establishment of marine generalist bacteria, which code for a large pool of functions. This is basically foreseen to cope with the heterogeneity of organic matter supplied to these systems. Nevertheless, it is not known what fraction of a generalist proteome is needed for house-keeping functions or what fraction is modified to cope with environmental changes. Here, we used high-throughput proteomics to define the proteome of Ruegeria pomeroyi DSS-3, a model marine generalist bacterium of the Roseobacter clade. We evaluated its genome expression under several natural environmental conditions, revealing the versatility of the bacterium to adapt to anthropogenic influence, poor nutrient concentrations or the presence of the natural microbial community. We also assayed 30 different laboratory incubations to increase proteome coverage and to dig further into the functional genomics of the bacterium. We established its core proteome and the proteome devoted to adaptation to general cellular physiological variations (almost $50 \%$ ). We suggest that the other half of its theoretical proteome is the opportunist genetic pool devoted exclusively to very specific environmental conditions.
\end{abstract}

The ISME Journal (2012) 6, 124-135; doi:10.1038/ismej.2011.86; published online 21 July 2011

Subject Category: integrated genomics and post-genomics approaches in microbial ecology

Keywords: proteomics; core proteome; specialist; generalist; Roseobacters; functional genomics

\section{Introduction}

Lifestyle dichotomies such as specialist/generalist or oligotroph/opportunitroph have been proposed to simplify the classification of microorganisms based on the adaptive strategies they apply in marine environments (Polz et al., 2006; Newton et al., 2010; Singer et al., 2011). Although specialist or oligotroph terms refer to microorganisms adapted to use low nutrient concentrations efficiently, generalists or opportunitrophs are predicted to be versatile microorganisms that are able to exploit variable resources spatially and temporally (Polz et al., 2006). Nevertheless, the genome usage of these organisms has not yet been clearly established. Oligotrophic oceanic waters are characterised by being largely colonised by specialised oligotrophic organisms, for example, the SAR11 cluster (Biers et al., 2009). A cultured representative of this group, Candidatus pelagibacter ubique, is a free-living microorganism with one of the smallest genomes

Correspondence: J Armengaud, Laboratoire de Biochimie des Systèmes Perturbés, CEA Marcoule, DSV, IBEB, SBTN, LBSP, F-30207 Bagnols-sur-Cèze, France.

E-mail: jean.armengaud@cea.fr

Received 29 March 2011; revised 30 May 2011; accepted 30 May 2011; published online 21 July 2011 known to date (Giovannoni et al., 2005). Despite this, it has the ability to use several different energy and carbon sources typically found in its environment. On the other hand, in surface and costal marine environmental niches, a higher predominance of bacteria with a generalist-like lifestyle is generally found, owing to the higher concentration of nutrients (Lauro et al., 2009) and greater heterogeneity of dissolved organic carbon in these waters (Mou et al., 2008). In agreement with the generalist lifestyle, microorganisms in these environments show no specialisation to a specific metabolic compound (Mou et al., 2008). Instead, bacteria adopt the strategy of accumulating a large pool of catabolic functions to face the diversity of dissolved organic carbon. To understand the functioning of marine communities and their impact on global ecosystem processes, numerous studies have been performed (reviewed in Strom, 2008; DeLong, 2009; Fuhrman, 2009). In order to help with the interpretation of marine metagenomic data and the physiology of relevant microbial groups, more than 150 marine bacterial genomes are currently being sequenced (http://www.moore.org). Despite this, little is known about what fraction of the encoded proteome has an impact on global ecosystem processes. Metaproteomics is becoming a key tool in revealing these functional profiles and determining 
the roles of each component in complex microbial communities (Wilmes and Bond, 2006, 2009; Keller and Hettich, 2009; VerBerkmoes et al., 2009).

The Roseobacter clade is a key component of surface marine ecosystems, as over $10 \%$ of bacterial cells in the open ocean and $20 \%$ in coastal waters are members of this group (Buchan et al., 2005). Their large inventory of encoded functions, in order to obtain carbon and energy, has established Roseobacters as generalists (Newton et al., 2010). Isolates show an enormously versatile metabolism, having important roles in the global carbon and sulphur cycles, and potentially modifying the climate, as they process important greenhouse gases such as carbon monoxide and dimethylsulphide (Wagner-Dobler and Biebl, 2006; Newton et al., 2010). Ruegeria pomeroyi DSS-3 (formerly Silicibacter pomeroyi) was the first member of the Roseobacter clade to have its genome completely sequenced and annotated (Moran et al., 2004). Inspection of the genome of $R$. pomeroyi revealed a wide gene pool, which is supposed to be helpful for adaptation to variations in the marine environment (Moran et al., 2004; Newton et al., 2010). Its genome comprises a 4.11-Mbp chromosome and a $0.49-\mathrm{Mbp}$ megaplasmid. This genome size is consistent with an generalist freeliving bacterial lifestyle with a large number of regulatory and signal transduction proteins (Koonin and Wolf, 2008). R. pomeroyi has become a model organism for studies of the ecological and physiological strategies of heterotrophic marine bacteria (Burgmann et al., 2007).

The large number of sequenced marine bacterial genomes has led to several comparative genomic studies (Moran et al., 2007; Scanlan et al., 2009). It is known that oligotrophic marine bacteria present 1.5-2 Mbp-sized genomes, whereas generalist genomes are much larger. Nevertheless, there is no experimental evidence of the fraction of their proteome needed for living in the different ecological niches found in the oceans. Interestingly, we have noted that genome-wide proteome analyses always show high coverage in oligotroph-like bacteria, for example, $88 \%$ coverage of the $777-\mathrm{Mbp}$ minimal organism Mycoplasma mobile (Jaffe et al., 2004), but much lower values when generalists are scrutinised, for example, $40 \%, 54 \%$ and $53 \%$ of the genes encoded by the 5.13-Mbp genome of Shewanella oneidensis, 3.4-Mbp genome of Sphingopyxis alaskensis and 3.9-Mbp genome of Synechocystis sp. PCC 6803, respectively (Gupta et al., 2008; Ting et al., 2009; Wegener et al., 2010). In the present study, we carried out a comprehensive proteomic analysis on a member of the marine Roseobacter clade that will give an overview of the functional genomics in this kind of organism. The proteomic patterns obtained after subjecting $R$. pomeroyi to different natural conditions showed only small variations, although these highlighted key proteins used by the bacterium in its environment. The extra 30 different physiological conditions tested enabled higher coverage of the organism's theoretical proteome. By comparing the high-throughput nanoliquid chromatography-mass spectrometry (LC-MS/ MS) proteomic data obtained throughout this study, we observed a large number of proteins detected under all conditions and those set for general physiological changes in the cell. Nevertheless, a larger number of proteins (over $50 \%$ of the theoretical proteome) were not detected, suggesting that this fraction is probably dedicated exclusively to allow adaptation to very specific physiological conditions or a generalist lifestyle.

\section{Materials and methods}

\section{R. pomeroyi culture conditions}

$R$. pomeroyi DSS-3 (DSM15171) was grown on marine agar plates (DIFCO/Becton Dickinson, Le Pont de Claix, France) before inoculating liquid cultures. This was performed using either marine broth (MB, Difco) or minimal marine medium (Lanfranconi et al., 2010). Different Marseille (France) coastal winter surface seawater samples were obtained to simulate natural conditions (2011-02-16): Bassin d'Arenc (ferry basin, 43.32567719N-5.35845279E); Vieux Port (marina, 43.29532433N-5.36682128E); Parc Borély (beach, 43.25770573N-5.37553310E); and Cap Croisette (cape, 43.21496607N-5.33602952E). The four different samples were processed immediately as follows: duplicated $750-\mathrm{ml}$ quantities of natural seawater (presence of the microbial community) or seawater filtered through a $0.22-\mu \mathrm{m}$-diameter pore filter (absence of the natural community) were placed in 1-l Erlenmeyer flasks. Each Erlenmeyer flask was inoculated with MB-pre-grown $R$. pomeroyi cells at a final concentration of $10^{5}$ cells $\mathrm{ml}^{-1}$. To avoid any addition of dissolved organic carbon to the seawater milieu, $R$. pomeroyi cells were washed twice with minimal marine medium before inoculation. The resulting 16 flasks (four different origins with the presence/absence of the natural microbial community, all duplicated) were agitated at 200 r.p.m. for $20 \mathrm{~h}$ at $25^{\circ} \mathrm{C}$. Similarly, community samples (without $R$. pomeroyi inoculum) were processed in order to assay for blanks, giving as a result no significant MS/MS-detected protein. For laboratory stress conditions, cells were grown and treated as shown in Table 1 and Supplementary Information. Whatever the conditions, cells were harvested by centrifugation for $10 \mathrm{~min}$ at $6000 \mathrm{~g}$ at $4{ }^{\circ} \mathrm{C}$. Pelleted cells were washed twice with cold $20 \mathrm{mM}$ Tris-HCl buffered at $\mathrm{pH}$ 8.0. Pellets were frozen at $-80^{\circ} \mathrm{C}$ until protein extraction took place. Protein extraction, SDS-PAGE and proteome fractionation are detailed in the Supplementary Information. Short electrophoresis migrations were carried out in order to detect the most abundant proteins and compare the different conditions assayed. To detect the lower abundant proteins, we simplified the bacterial protein mix with long electrophoresis migrations. 
Table 1 Laboratory growth and stress conditions applied to Ruegeria pomeroyi

\begin{tabular}{|c|c|c|c|c|c|}
\hline Sample & Duplicated & Medium & Incubation/stress ${ }^{\mathrm{a}}$ & Growth stage ${ }^{\mathrm{b}}$ & Other details \\
\hline C01 & $A$ and $B$ & MB & & Exponential phase & 1 day until peak growth \\
\hline C02 & & MB & & Late stationary phase & 1 day until peak growth \\
\hline C03 & $A$ and $B$ & MMM YE succinate & & Exponential phase & 2 day until peak growth \\
\hline C04 & & MMM YE succinate & & Late stationary phase & 2 day until peak growth \\
\hline C05 & & MA & Cells grown on agar plates (3 days) & Stationary phase & \\
\hline C06 & A and $B$ & MA & Cells grown on agar plates (10 days) & Late stationary phase & \\
\hline C07 & & $\mathrm{MB}$ & Microaerobic conditions & Early stationary phase & $\begin{array}{l}5 \text { days until peak growth } \\
\left(\mathrm{OD}_{\text {60о }} 0.4\right)\end{array}$ \\
\hline C08 & & MB & $40^{\circ} \mathrm{C}$ & Mid stationary phase & 4 days until peak growth \\
\hline C09 & & MB & $12^{\circ} \mathrm{C}$ & Early stationary phase & 3 days until peak growth \\
\hline C10 & & MB & $7.5 \%$ sea salts & Mid stationary phase & 4 days until peak growth \\
\hline C12 & & MB & UV stress (15s) & Mid stationary phase & $2 \%$ CFU survival \\
\hline C13 & & MB & UV stress (1 min) & Mid stationary phase & $0,2 \%$ CFU survival \\
\hline C15 & $A$ and $B$ & MB & UV stress (5 min) & Mid stationary phase & $0,02 \%$ CFU survival \\
\hline C16 & & MB grown cells ${ }^{\mathrm{c}}$ & Pelleted cells incubated at $26^{\circ} \mathrm{C}$ & Stationary phase & 1 day incubation \\
\hline C17 & & MB grown cells ${ }^{\mathrm{c}}$ & Pelleted cells incubated at $4{ }^{\circ} \mathrm{C}$ & Stationary phase & 1 day incubation \\
\hline C18 & & MB & $0.2 \%$ sea salts & Early stationary phase & 5 days until peak growth \\
\hline C19 & & MB grown cells ${ }^{\mathrm{c}}$ & Incubated in Tris- $\mathrm{HCl} 20 \mathrm{mM}$ pH 8.2 & Stationary phase & 1 day incubation \\
\hline $\mathrm{C} 21$ & & MMM succinate & No YE added & Mid stationary phase & $\begin{array}{l}4 \text { days until peak growth } \\
\left(\mathrm{OD}_{600} 0.2\right)\end{array}$ \\
\hline C25 & & MB grown cells ${ }^{\mathrm{c}}$ & Incubated in autoclaved SW & Stationary phase & 1 day incubation \\
\hline $\mathrm{C} 28$ & & SW succinate & & Mid stationary phase & 4 days until peak growth \\
\hline C31 & & MB-grown cells ${ }^{\mathrm{c}}$ & Incubated in MMM naphthalene $0.5 \%$ & Stationary phase & 1 day incubation \\
\hline C32 & & $\mathrm{MB}$ & Diesel $0.3 \mathrm{ml} \mathrm{l}^{-1}$ & Early stationary phase & 1 day until peak growth \\
\hline C33 & & MB & Diesel $3 \mathrm{ml} \mathrm{l}^{-1}$ & Early stationary phase & 1 day until peak growth \\
\hline C34 & & MB grown cells ${ }^{\mathrm{c}}$ & High cell concentration $\left(10^{11} \mathrm{CFU} \mathrm{ml}^{-1}\right)^{\mathrm{d}}$ & Stationary phase & $\begin{array}{l}1 \text { day incubation in high } \\
\text { density cell suspension }\end{array}$ \\
\hline C35 & & MB & Diesel $30 \mathrm{mll}^{-1}$ & Early stationary phase & 1 day until peak growth \\
\hline C36 & & MMM YE Benzoate & & Mid stationary phase & 4 day until peak growth \\
\hline C38 & & $\mathrm{MB}$ & Naphthalene $0.5 \%$ & Mid stationary phase & $\begin{array}{l}4 \text { day until peak growth } \\
\text { (brown culture) }\end{array}$ \\
\hline C41 & & MB & UV stress (15s) & Early stationary phase & $3 \%$ CFU survival \\
\hline $\mathrm{C} 43$ & & MB & Heat shock $\left(42^{\circ} \mathrm{C} ; 30 \mathrm{~min}\right)$ & Early stationary phase & $30 \%$ CFU survival \\
\hline C46 & & MB & Cold shock $\left(-20^{\circ} \mathrm{C}\right.$ until freezing $)$ & Early stationary phase & $80 \%$ CFU survival \\
\hline
\end{tabular}

Abbreviations: MA, marine agar; MB, marine broth; MMM, minimal marine media; SW, sea water; YE, yeast extract.

${ }^{a}$ If not mentioned incubation/stress was carried out at $26^{\circ} \mathrm{C}$ under agitation at $180 \mathrm{r}$.p.m. $R$. pomeroyi is unable to grow using naphthalene or diesel as unique sources of energy and carbon, hence their inclusion in this column.

bexponential phase $\left(\mathrm{OD}_{600}\right.$ 0.7), early stationary phase (when culture reached peak growth), mid and late stationary phase (1 and 3 days, respectively, after the culture reached stationary phase).

c'MB grown cells' is indicated under those conditions where $R$. pomeroyi was not able to grow due to the lack of a useful source of energy and carbon. Cells had to be inoculated in sufficient number for the MS/MS analysis after the incubation/stress.

${ }^{\mathrm{d}} \mathrm{A} 100 \mathrm{ml}$ grown culture was centrifuged and the pellet was resuspended in $1 \mathrm{ml}$ of the remaining MB supernatant.

Trypsin in-gel proteolysis, nano-LC-MS/MS, database searches and spectral count

Polyacrylamide gel bands were cut and processed for in-gel proteolysis with trypsin (Roche, Meylan, France) following the ProteaseMAX protocol (Promega, Charbonnières-les-Bains, France) as described (Clair et al., 2010). Nano-LC-MS/MS experiments were performed using the LTQ-Orbitrap XL hybrid mass spectrometer (ThermoFisher, Courtaboeuf, France) coupled to an UltiMate 3000 LC system (Dionex-LC Packings, Voisins le Bretonneux, France) as previously described (de Groot et al., 2009; Baudet et al., 2010). Database and peak list generation, as well as the parameters for MS/MS database, are described in the Supplementary Information searches. Protein semi-quantitation by spectral abundance took place as previously described (Liu et al., 2004). For determining the normalised spectral abundance factors of each protein, spectral counts assigned to each polypep- tide were divided by its molecular weight (Paoletti et al., 2006). Values were then normalised by the total sum corresponding to all the polypeptides detected with two or more non-redundant peptides. Clustering and sequence analysis is detailed in the Supplementary Information. The statistical protein variation among the duplicated samples of the natural conditions was calculated with the ACFold option of the PatternLab software (Carvalho et al., 2008). This module combines average fold changes with statistics (AC-test) and a theoretical false-positive estimator to properly select the differentially expressed proteins. We chose stringent parameters: significance threshold at $P<0.01$ and fold change $>2.0$. Protein levels detected in cells subjected to laboratory conditions (without duplicates) were compared with the mean levels of these proteins considering all the conditions. In this case, only fold changes $>4.0$ were considered. 


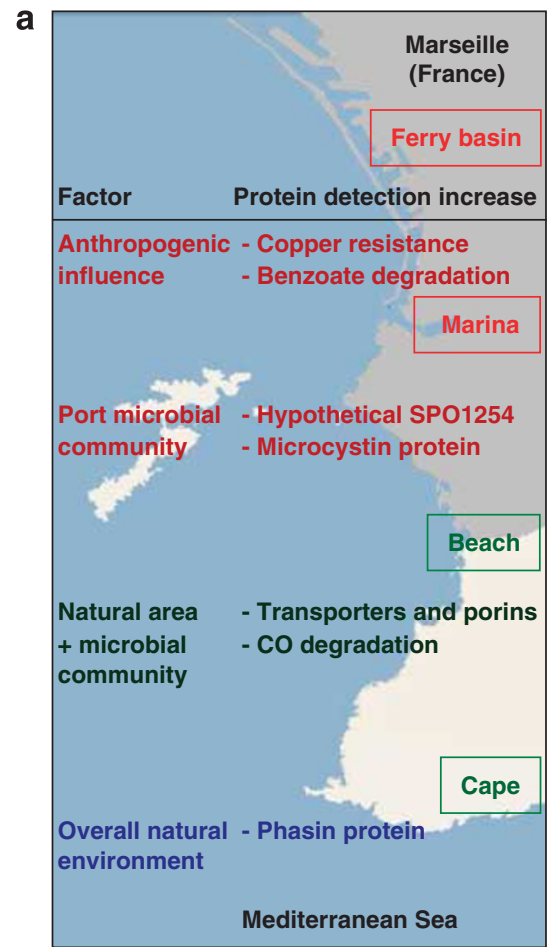

b

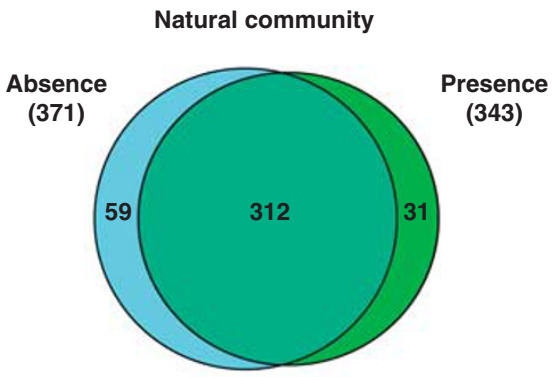

Anthropogenic influence

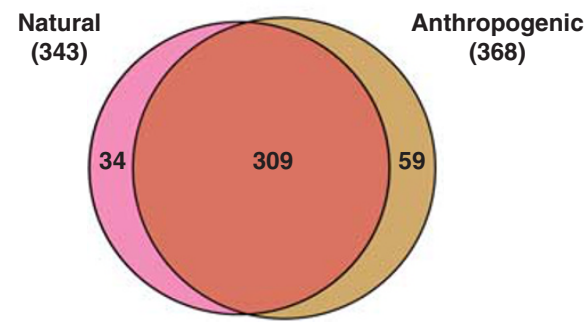

Figure 1 Polypeptides detected from $R$. pomeroyi cells incubated under different natural conditions. (a) Sampling areas where the seawater for natural-condition simulation was obtained. Anthropogenically perturbed and natural areas are in red and green, respectively. The most statistically differing proteins detected in $R$. pomeroyi in each environmental condition compared with other conditions are indicated (statistical analysis shown in Supplementary Table S2). 'Anthropogenic influence' represents the main protein differences between the ferry basin and marina to cape conditions (in the presence and absence of the natural community); 'Port microbial community' compares the overproduced proteins of $R$. pomeroyi in the presence of the natural community in both port systems; 'Natural area + microbial community' highlights those proteins overproduced in the cape and beach conditions with the natural community when compared with the port systems; 'Overall natural environment' compares the natural conditions tested (polypeptide list of Supplementary Table S1) with the laboratory conditions assayed (polypeptide list of Supplementary Table S3). (b) Both Venn diagrams show the number of shared and specific polypeptides detected in the absence and presence of the natural community (top) and based on the anthropogenic influence (bottom). For this, the data were grouped and compared as follows: the four sampling areas in the absence (blue) versus presence (green) of the natural community; natural (beach and cape in the presence/absence of the natural community, pink) versus anthropogenicallyperturbed (marina and ferry basin in the presence/absence of the natural community, brown) seawater areas. In brackets are the total non-redundant polypeptides corresponding to each group.

\section{Results}

Proteome variations of $\mathrm{R}$. pomeroyi in its natural coastal environment

Cells of $R$. pomeroyi were incubated in natural seawater taken from four different sampling areas (open cape, beach, ferry basin and marina) in the presence or absence of the natural microbial community (Figure 1a). R. pomeroyi cells were inoculated at a concentration of $10^{5}$ cells $\mathrm{ml}^{-1}$. Cells from each of the duplicated culture conditions were harvested $20 \mathrm{~h}$ later. Their protein contents were analysed by means of shotgun nano-LC-MS/MS proteomic analysis in order to detect only the more abundant proteins of $R$. pomeroyi (Supplementary Table S1). We defined the abundant proteins as those accounting for at least $50 \%$ of the total quantities (here estimated with spectral count) detected in an exhaustive proteomic analysis (when considering the most minimal set). Figure $1 \mathrm{~b}$ shows that the presence of the microbial community or the origin of the seawater does not result in a strong variation in the detected abundant-protein pattern of $R$. pomeroyi. Nevertheless, the number of proteins with a significant variation is slightly higher when the seawater origin is considered (Supplementary Table S2). It is noteworthy that no significant falsepositive attributions were obtained from the natural community samples (blank samples detailed in the Materials and methods section).

Protein profiling in the presence/absence of the microbial natural communities. In the natural seawater samples (cape and beach), where microbial density and nutrient concentration are expected to be lower, we found no significant protein variation when $R$. pomeroyi was incubated in the presence or absence of the natural community $(P<0.01$, Supplementary Table S2, panels A and B). Nevertheless, we noted that transporters and porins were possibly more abundant in cape water when the natural community is present (fold change 2-5 $\times$ ), but with a low statistical confidence $(P$-value $<0.06)$, plausibly due to competition for nutrients. Meanwhile 


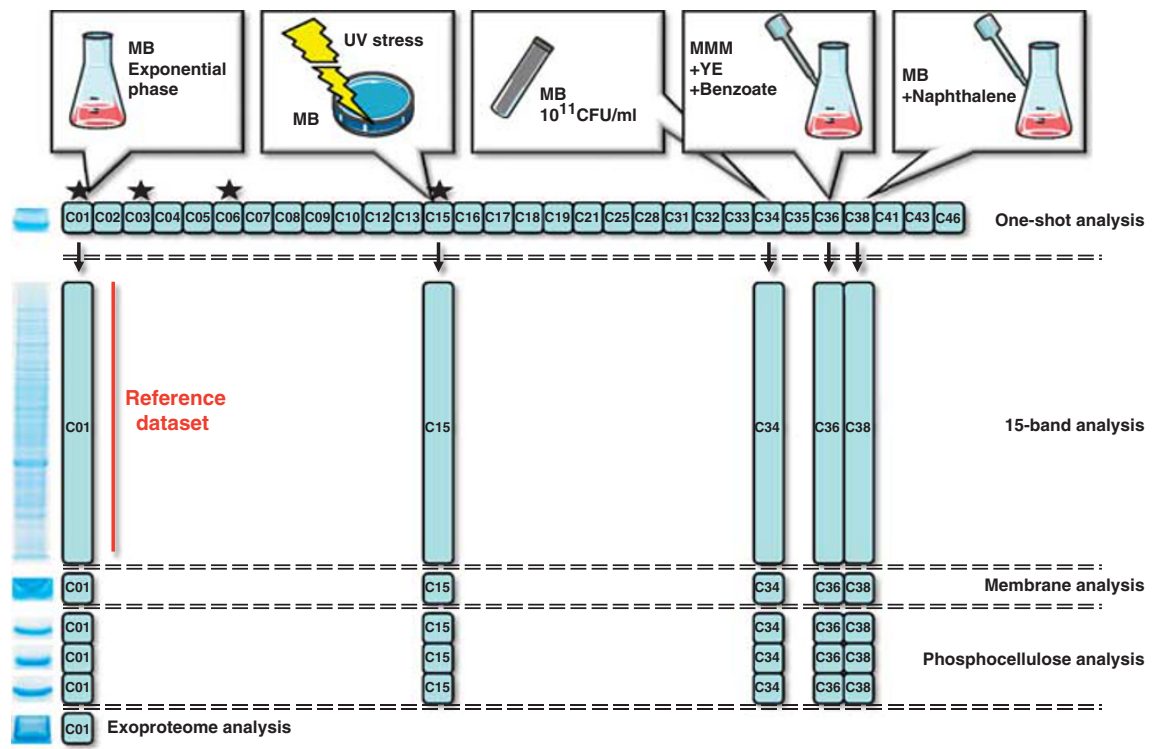

Figure 2 Strategy used to obtain the broadest proteome coverage for $R$. pomeroyi. An image of the Coomassie-stained SDS-PAGE gel is shown for the reference data set (far left) to illustrate the extent of migration. A star indicates those conditions for which biological and technical duplicates were performed. Conditions are numbered from C01 to C46 as indicated in Table 1.

this did not happen in the anthropogenically perturbed seawater samples (ferry basin and marina), where transporters are less detected with the presence of the natural community (Supplementary Table S2, panels $\mathrm{C}$ and D). Two proteins were significantly more abundant in the presence of port communities: the hypothetical orfan protein SPO1254 (fold change $16 \times$ ) and the microcystindependent protein SPO0483 $(10 \times)$ that presents a conserved phage tail collar domain, which could be involved in pathogenicity or plant-microbe interactions.

Protein profiling between the different sampling areas. Beach, ferry basin and marina samples were compared with the cape samples in the absence (Supplementary Table S2, panels E-G) and presence of the natural community (Supplementary Table S2, panels H-J). The plausible higher concentration of nutrients in the anthropogenically perturbed seawater samples may be the reason for the observed decrease in the abundance of many transporters in these conditions. A strong increase (between 12-35 $\times$ ) in copper resistance proteins (SPOA0369 and SPOA0370) was evidenced in both port samples. This could be a consequence of the environmental use of copper antifouling paints for boat hulls. The benzoyl-coA oxygenase (SPO3701) detected in the marina samples $(4 \times)$ was also remarkable and probably illustrates anthropogenic influence in these environments. We also found that $R$. pomeroyi has a lithotrophic lifestyle, where dissolved organic carbon is possibly less abundant (that is, cape seawater samples), through the detection of carbon monoxide degradation determinants. Another interesting feature of $R$. pomeroyi in its natural environment is the production of phasin, a protein involved in the accumulation of polyoxoesters (York et al., 2001). This protein is not usually detected under laboratory conditions (see below).

\section{Proteomic-based screening for the most divergent laboratory conditions}

Figure 2 shows the strategy followed to determine the most divergent physiological conditions for $R$. pomeroyi in terms of its proteome. Briefly, we assayed the most abundant proteins present in cells grown under 30 different laboratory conditions by means of short-migrated 1D-gel stacking. As shown in Table 1, we tried out exponential and stationary culture phases in rich and poor media, with different incubation parameters (salt concentration, temperature, microaerobiosis, pelleted cells and plate growth) and aggressive stresses (UV illumination, the presence of aromatic compounds). Biological (A and B) and technical (B1 and B2) duplicates were performed for four conditions to estimate the variability of our experimental procedure. A total of 878 non-redundant proteins were catalogued by their abundance in the whole screening (Supplementary Table S3). A hierarchical cluster analysis revealed the relationships between the protein profiles from the 30 conditions assayed (Figure 3). As expected, the 8 samples that corresponded to either technical or biological replicates grouped together. Furthermore, we chose the five growth conditions that generated the most divergent proteomes for an in-depth analysis (Figures 2 and 3). We resolved the total extracted proteins on a longergel electrophoresis run and analysed 15 fractions. We also enriched lower abundant proteins such as 


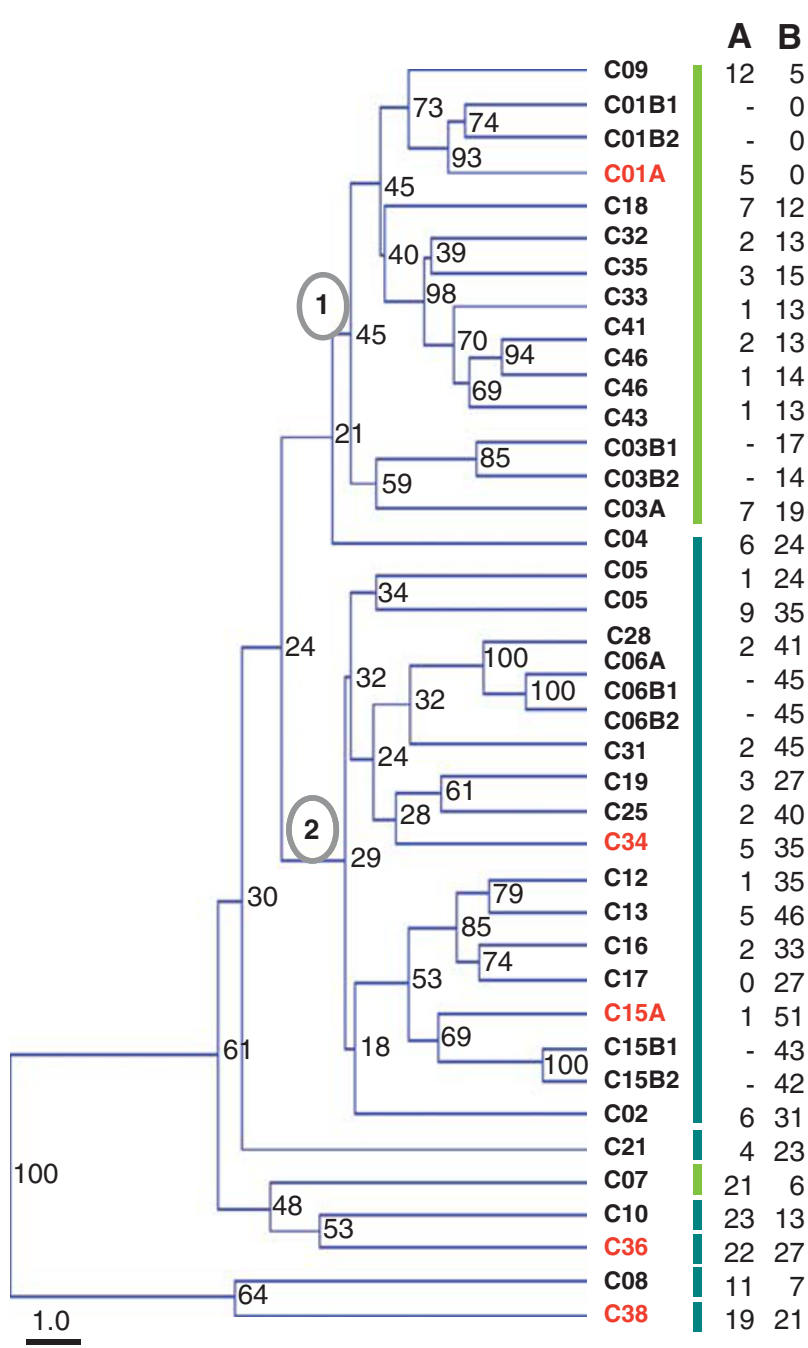

Figure 3 Hierarchical clustering of the protein profiles corresponding to 30 different growth conditions and 8 replicates based on MS/MS one-shot analysis. The tree was produced using UPGMA and Euclidean distance analysis. Bootstrap values (1000 replicates) are indicated. Clusters $\mathbf{1}$ and $\mathbf{2}$ indicate protein profiles grouped according to growth phase, exponential-early stationaryphase and mid-late stationary-phase, respectively. Light green lines indicate exponential-early stationary-phase cultures, while dark green lines indicate mid-late stationary-phase cultures. The number of detected and validated polypeptides that are unique to each condition compared with all the others is given in column A. Column B represents the polypeptides not seen in the reference dataset (C01 15-band analysis).

phosphate-binding proteins (phosphocellulose P11 chromatography) and membrane proteins, and included data from its exoproteome (Christie-Oleza and Armengaud, 2010). Supplementary Table S4 compiles all the non-redundant peptides and proteins detected by MS/MS under the five conditions. From the 831851 spectra recorded in the whole study, a total of 1963 polypeptides (Supplementary Table S5) were detected with high confidence (global false positive rate of $1.2 \%$ ) covering $46 \%$ of the theoretical $R$. pomeroyi proteome (4252 annotated CDS).
Proteomic trends among the different physiological conditions

General protein pattern detected between the physiological conditions tested. Ribosomal proteins represented over $26 \%$ of the normalised spectral abundance factor for the total proteins detected from cells obtained from exponential and early stationary phase conditions, but only $14 \%$ in those harvested at the mid-late stationary-phase. The abundance of these proteins is directly related to cellular activity. For this reason, protein profile grouping related to the culture growth phase (exponential-early stationary (1) versus mid-late stationary (2) phases in Figure 3) prevails over the other parameters. Two main branches, which group 24 of the 30 conditions, clearly correspond to the distinct growth phases. The profile of condition C04 is the only stationaryphase growth that groups slightly with the exponential phase cluster, probably owing to the similarities to condition $\mathrm{C03}$ and to a plausible metabolic 'stand-by' effect already reported in other Roseobacter members (Zech et al., 2009). This adaptation strategy is foreseen to maintain central carbon catabolism unchanged during the initial transition from exponential to stationary growth phase. In contrast, the presence of the aromatic compound naphthalene (C38), high salt concentrations (C10), $\mathrm{O}_{2}$ limitation (C07), high temperatures (C08) or growth using benzoate (C36), are conditions that present the most protein differences. In these cases, proteins abundantly produced to cope with the corresponding stresses prevail over the protein pattern changes due to culture phase transition.

We noted the presence of the hypothetical protein SPOA0343 among the most detected proteins under almost all the growth conditions tested, except those grouping outside clusters 1 and 2 (Supplementary Table S3). Figure 4 shows the levels of detection for the proteins coded by the SPOA0339-SPOA0340SPOA0341-SPOA0342-SPOA0343 genes, which form a probable operon on the pDSS-3 megaplasmid. The products of the two genes SPOA0342 and SPOA0341 (annotated as hypothetical genes), and of SPOA0340 and SPOA0339 (with a functional annotation as a putative tautomerase and a HAD family hydrolase, respectively) were also detected. As SPOA0342 is a distantly related homologue of the AfsA protein from Streptomyces griseus (Kato et al., 2007) involved in A factor biosynthesis, we may infer a role in microbial hormone biosynthesis for this plausible operon. Such a role is in agreement with the fact that detection of these five proteins strongly decreases under adverse stress conditions.

Specific proteins overproduced under the different physiological conditions tested. We observed some differences according to whether the cells had to face a sudden heat shock or whether they were allowed a longer adaptation time to high temperatures. On the one hand, under conditions C08 and C43 (growth at $40^{\circ} \mathrm{C}$ and heat shock at $42^{\circ} \mathrm{C}$, 
130

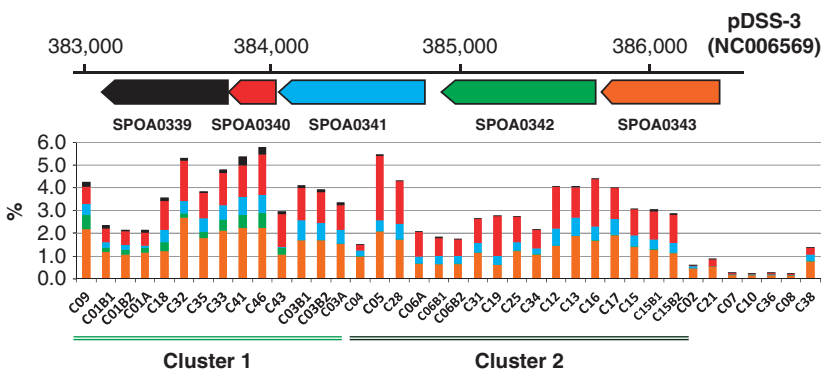

Figure 4 Detection of proteins SPOA0339-SPOA0340SPOA0341-SPOA0342-SPOA0343 among the different physiological conditions tested. The putative operon structure on the pDSS-3 megaplasmid is shown. The stacked bar graph shows the accumulation of normalised spectral abundance factor values for the five detected proteins under each of the 30 conditions and eight replicates. Conditions are sorted as in the hierarchical clustering shown in Figure 3. Clusters 1 and 2 are indicated.

respectively) we observed a response probably to avoid protein denaturation and aggregation that comprises the ClpB chaperone (SPO3276, 15×

higher than the average of detection under all conditions) and the La protease (SPO2613, $7 \times$ ). On the other hand, for long-term adaptation to high temperatures (only under condition C08), $R$. pomeroyi produces the GroEL chaperonin (SPO0887, $4 \times$ ), a membrane integrated protein with lipid raft-associated functions (SPO2617, $13 \times$ ), and a hypothetical transmembrane protein (SPO2567, $11 \times$ ). At low temperatures (C09, growth at $12^{\circ} \mathrm{C}$ ), $R$. pomeroyi increases the production of specific proteins: the cold shock protein CspA (SPO3625, 18 $\times$ ), the DEAD-box helicase (SPO3833, 20×), which increases the cold tolerance of transcriptional regulators CBF/DREB, the transcriptional regulator CarD (SPO1420, $7 \times$ ), the elongation factor TypA $($ SPO2031, $10 \times)$ and the FKBP isomerase involved in protein folding (SPOA0438, $8 \times$ ). The cellular responses observed under these temperature-related conditions are highly consistent with those described for another marine bacterium, Sphingopyxis alaskensis (Ting et al., 2009). At high salt concentrations near $R$. pomeroyi's growth limit (condition C10, $7.5 \%$ sea salts), a Na-translocating complex comprising SPOA0028 $(14 \times)$, SPOA0029 $(7 \times)$ and SPOA0030 $(9 \times)$ is overproduced. When $R$. pomeroyi is grown in natural sea water supplemented with $0.5 \%$ succinate (C28), nitrogen-regulatory proteins P-II (SPO2294, $11 \times$ and SPO3724, 16 $\times$ ) and nitrogen-regulation protein $\mathrm{NtrC}$ (SPO2087, $9 \times$ ) have a higher detection rate than under any other conditions. These proteins are probably overproduced to cope with an expected nitrogen imbalance in this environment after adding an excess carbon source. In condition C28, we also noted an accumulation of the phasin protein $(8 \times)$ as reported in the natural seawater conditions described here, SPO1710 (the periplasmic component of an active transport system for short-chain amides and urea, $19 \times$ ) and SPO2261 (an HK97 capsid-like protein,
$11 \times)$. On the other hand, when comparing agar plate conditions (C5 and C6, plausible simulation of biofilm formation) to their respective liquid culture conditions (C01 and C02), we observed a decrease in the levels of detection of RNA polymerase $(6 \times$ for SPO3507 and SPO3508), ribosomal proteins (from $26 \%$ to $13 \%$ ) and central metabolic enzymes, typical of stationary-phase cells, and a steep increase in carbon monoxide dehydrogenase (SPO1519 $44 \times$ and SPO1520 $16 \times$ ) and metalo-beta-lactamase enzyme levels (SPO0429 $9 \times$ and SPO2170 $24 \times$ ).

It is also interesting to highlight those proteins that were overproduced in the presence of diesel and naphthalene. Although it has not been demonstrated that $R$. pomeroyi can assimilate or mineralise these compounds, several proteins are detected as overproduced. They may be involved in the bacterium response to the plausible toxic effects of these compounds using mechanisms as reviewed by Ramos et al. (2001). R. pomeroyi tolerates high concentrations of diesel added to MB (C35, 3\% of diesel) well, as shown by its normal growth (1 day until peak growth) such as that seen under condition C01 (Table 1). Based on the proteomic results, addition of diesel gives rise to several similar processes to those observed under the microaerobic condition C07. We noted a likely increase in nitrogen reduction triggered by the production of SPOA0050 (a nitrous-oxide reductase, $9 \times$ ) and SPOA0220 (a cytochrome cd1 nitrite reductase, $12 \times)$, as well as a need for ATP synthesis from ADP with the production of polyphosphate kinase 2 (SPO1256, $8 \times$ ) to cope with a plausible lower redox potential. In this sense, diesel seems to limit oxygen exchange, producing a microoxigenic state for the bacterium. The presence of diesel also requires membrane stabilisation with the production of the transmembrane protein SPO2567 (highly detected under naphthalene condition C38, see below) and with the biogenesis of rhamnose for the outer cellular envelope (SPO0212, 16 $\times$ ). On the other hand, naphthalene seems to have a much more toxic effect on $R$. pomeroyi (4 days until peak growth, Table 1). In the presence of this aromatic compound, membrane proteins that could have a role in stabilising the cell membrane, SPO2567 $(13 \times)$, SPO2568 $(8 \times)$ and SPO3325 $(11 \times)$, were highly detected. These proteins were also strongly produced under condition C08 (growth at $40{ }^{\circ} \mathrm{C}$ ). We also detected strong production of proteins SPO2757 (EF hand domain-containing protein, $9 \times$ ), SPO0115 (bifunctional enoyl-CoA hydratase/ phosphate acetyltransferase, $14 \times$ ) and SPO1625 (periplasmic serine protease, $19 \times$ ) that have plausible detoxifying roles, and three proteins from efflux pumps SPO1497 $(16 \times)$, SPO0111 $(16 \times)$ and SPO1094 $(12 \times)$.

Differential protein detection among the five conditions used for in-depth analysis. Proteins related to translation, ribosomal structure and 
a
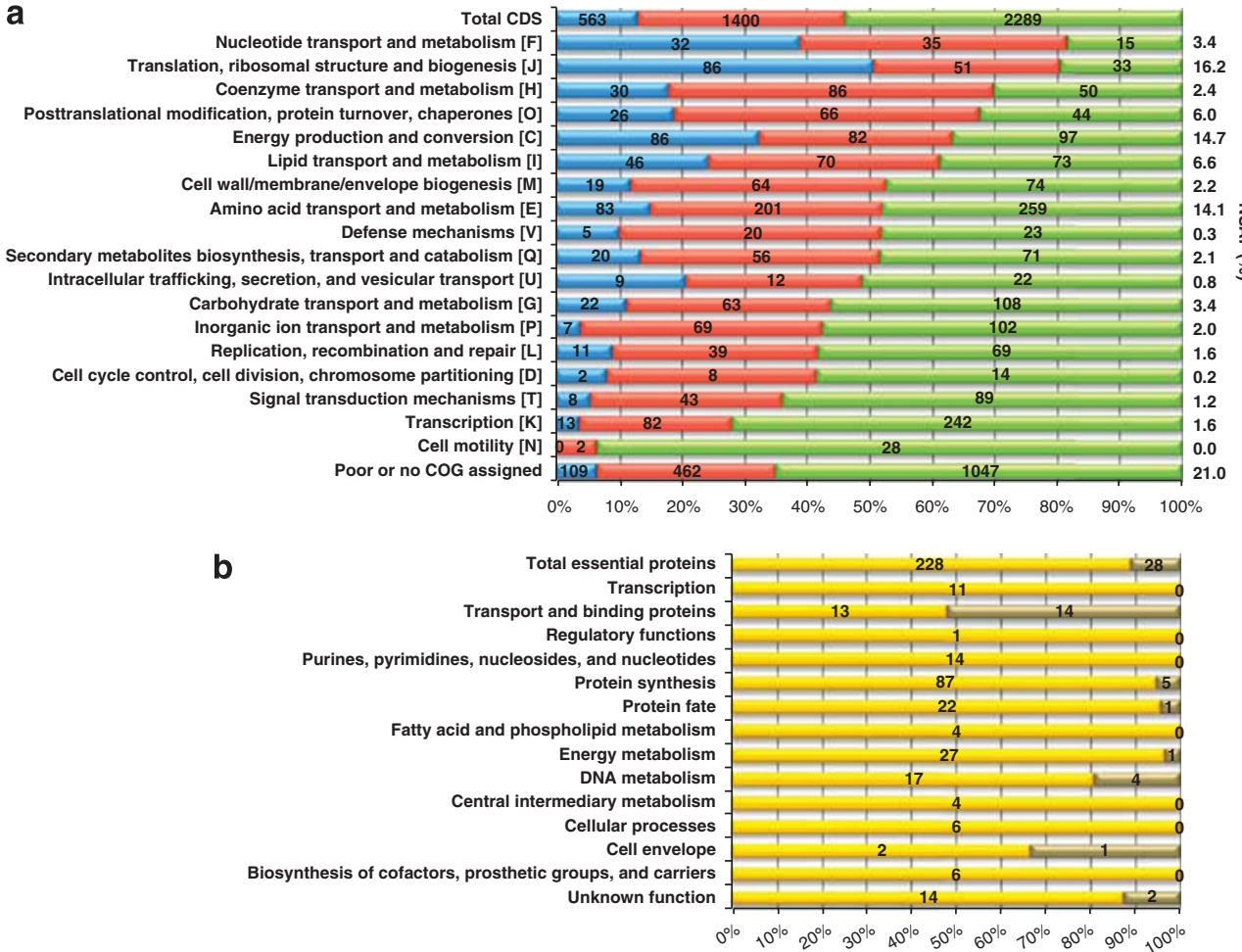

Figure 5 Total and putative essential proteome from $R$. pomeroyi sorted by functional categories. Total proteins in each category are graphed by $100 \%$ stacked bars. (a) Proteins belonging to the detected core proteome (blue), accessory-detected proteome (red) and nondetected proteome (green) for each of the COG categories. (b) Detected (yellow) and non-detected (grey) proteins of the putative essential proteome of $R$. pomeroyi sorted by the categories assigned by Glass et al. (2006). The number of proteins corresponding to each of the bars is shown. The normalised spectral abundance factor (NSAF) values are the accumulated protein abundance values observed in the 5 in-depth 15-band analyses (Supplementary Table S4).

biogenesis (16\%), energy production and conversion $(15 \%)$, and amino-acid transport and metabolism $(14 \%)$ were among the most abundant proteins detected irrespective of the growth conditions (Figure 5). Interestingly, the four conditions that had entered the stationary phase (C15, C34, C36 and C38) presented a considerable increase in transporters for the uptake of nutrients remaining in the medium: 98 polypeptides detected in C34 (14\% of the total normalised spectral abundant factor), while only 55 in C01 ( $<2 \%$ of the total). Again, the overall relative abundance of ribosomal proteins decreased slightly under all stationary conditions (8-12\%) when compared with the exponential phase sample C01 (18\%). The transmembrane protein SPO2567 commented above represented over $19 \%$ of the proteins detected during growth in the presence of naphthalene (C38), while it was detected in much lower quantities $(0.02-0.34 \%)$ under the other four conditions. As $R$. pomeroyi shows a slower growth in the presence of naphthalene, we may suggest a possible function related to specific stress tolerance or membrane stabilisation for this protein. Homologues of SPO2567 are systematically found in 29 of the 32 sequenced Roseobacter genomes currently deposited in the Roseobase database. We also noted that SPO2568, annotated as 'hypothetical protein' and encoded in the close neighbourhood of the
SPO2567 gene, has a similar detection profile. Other proteins also became abundant in specific samples, which were consistent with the growth conditions applied. For example, benzoate degradation enzymes that are coded in a probable operon (benzoate-coA ligase, benzoate-coA oxygenase A and $\mathrm{B}$ subunits, hydrolase and an enoyl-coA hydratase) were abundantly detected only under condition C36. Stress response proteins were observed under condition C15, that is, two different polypeptides, namely SPO3527 and SPO2371, annotated as universal stress proteins. These polypeptides have an Usp-like conserved sequence that corresponds to a putative nucleotide binding domain and may carry out a function resulting in enhancing cellular survival in the presence of stress agents (Gustavsson et al., 2002).

\section{Products of putative essential genes and core MS-detected proteome}

In the overall analysis, the COG 'nucleotide transport and metabolism' and 'translation, ribosomal structure and biogenesis' groups displayed the highest detection ratio (Figure 5a). On the other hand, proteins from 'signal transduction mechanisms' and 'transcription' (generally transcriptional regulators) COG categories were less detected and found to be 
condition-specific. We analysed whether the products of already known essential genes in bacteria were listed in our MS/MS-detected catalogue. For this, we identified $R$. pomeroyi proteins that are homologues to the products of the 382 genes listed as essential in Mycoplasma genitalium (Glass et al., 2006) by PSI-BLAST and manual curation. Supplementary Table S6 lists the 256 genes that could be confidently identified in the $R$. pomeroyi genome on this basis. A total of $228(89 \%)$ of the corresponding proteins were detected by MS/MS (Figure 5b). The only abundant group of proteins that presented a low detection ratio (48\%) was the one corresponding to 'transport and binding proteins'. This was expected owing to the exclusive growth conditions applied to M. genitalium (Glass et al., 2006) and the wide range of $\mathrm{ABC}$ and TRAP transporters observed in $R$. pomeroyi (Moran et al., 2007). In the whole study, 145 polypeptides related to these transporters were detected, out of a total of $368(39 \%)$ annotated in the genome. Excluding this specific group of proteins from the putative essential gene analysis, we covered $94 \%$ of the total essential genes (215 of the 229) within the whole detected proteome reported here.

The proteins were systematically detected, no matter what culture conditions were listed and designated as the core MS-detected proteome. A total of 563 polypeptides were found (shaded items in Supplementary Table S5) when compiling those detected under all 5 conditions analysed in depth. This core MS-detected proteome could define the main physiological basic traits of $R$. pomeroyi. Indeed, we observed a high predominance of proteins belonging to the 'translation, ribosomal structure and biogenesis', 'energy production and conversion' and 'amino-acid transport and metabolism' COG groups. From the core-proteome list, 318 proteins were found to be strictly conserved (sequence similarities with $E$ values below $10^{-20}$ ) out of the 32 currently sequenced Roseobacter genomes. This number notably increases when considering a less strict degree of occurrence, this being 481 polypeptides conserved in 27 out of 32 genomes (Supplementary Table S5). Interestingly, 48 'hypothetical' proteins were evidenced in this core MS-detected proteome. Their function should be important as they are detected systematically under all 5 conditions. Of these proteins, 31 are relatively well conserved among the 32 sequenced Roseobacter genomes (seen in over 23 of these isolates), whereas 5 are exclusive to $R$. pomeroyi (SPOA0341 and SPOA0343, two proteins with a plausible role in hormone production commented above, SPO1254, SPO1996 and SPO2776). When comparing the MSdetected core proteome of $R$. pomeroyi with the Roseobacter core genome defined previously by Moran et al. (Moran et al., 2007), we found $66 \%$ of the MS-detected proteins (370 out of 563) among the 1310 proteins established by comparative genomics (Supplementary Table S5). When comparing our core proteome with a more recent genomic analysis of marine Roseobacters that restricted the coregenome set (Tang et al., 2010) to 694 items, we found $68 \%$ (475) of these as MS-detected in our whole study.

\section{Confines of identifiable proteome}

Figure 6 shows the protein discovery saturation curves in terms of novel detected proteins as we added new growing conditions up to 30 (panel a) and the 5 conditions analysed in depth (panel b). In both cases, the rapid increase in terms of identification of proteins observed after analysing the first conditions was followed by apparent non-saturation. This indicates that under each specific condition assayed, a few novel genes were being expressed in order for the bacterium to cope with the novel environment conditions or stress. Figure 6c, shows that for abundant proteins, identification seems to saturate below 1500 polypeptides if the logarithmical tendency is extrapolated to 1000 theoretically different physiological conditions. Remarkably, the limit would be the total theoretical proteome $(4252$ polypeptides) through a 15-band fully resolved gel-based approach (Figure 6d). Although the data correlate well with a logarithmic regression tendency ( $r^{2}$ is over 0.99 in both cases), these considerations should be taken with caution, as experimental data do not cover the inflection point of the curve. At this stage, another aspect to take into consideration is when novel polypeptide detection overlaps false positive increase (Figure 6e). In this case, the powered algorithm tendency of novel polypeptide detections among the total and the rate of predicted false positive accumulation is shown. The false positive detection would exceed novel polypeptide detection after analysis of 38 in-depth conditions, being in this case above $5.2 \%$ (Figure 6e).

\section{Discussion}

Here, we present the first study describing the proteome variations of a marine bacterium in its natural environment and the influence exerted by the natural community. The four different seawater samples tested in the presence or absence of a natural seawater microbial community revealed interesting variations regarding the production of several key proteins (Figure 1a). Nevertheless, most of the proteins detected (over 95\%) showed no significant variation between the different conditions compared. In order to stress the limits of the expressed genome of the bacterium we processed 30 different laboratory conditions. This allowed us to establish the broadest proteome compilation of a $R$. clade member, covering $46 \%$ of its total theoretical proteome. We confidently validated $1963 R$. pomeroyi DSS-3 polypeptides after detecting 23957 nonredundant peptides by MS/MS. In this way we 

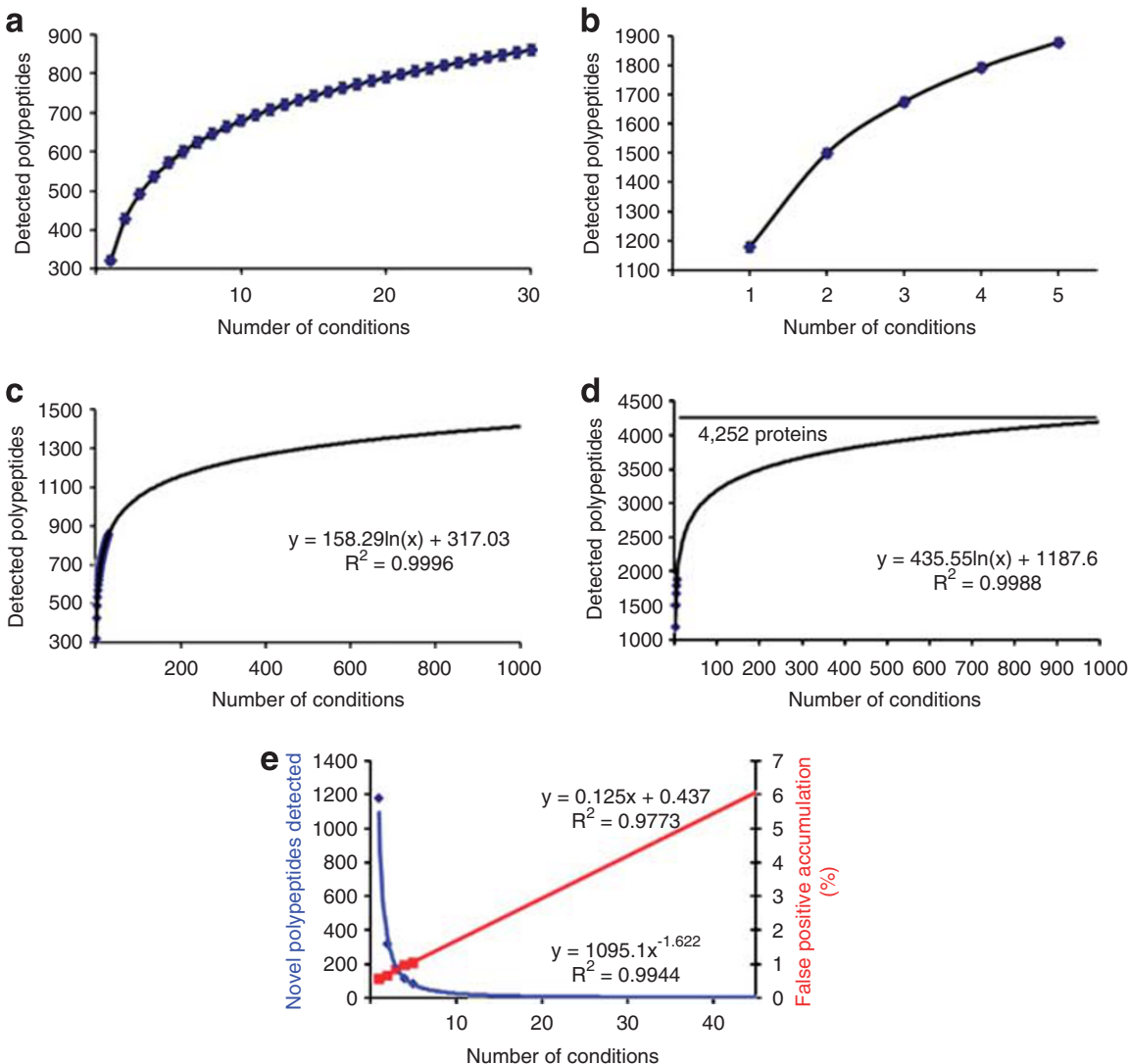

Figure 6 Protein discovery saturation curves. Polypeptide accumulation among the 30 growth conditions (a) and 5 in-depth analyses including 15-band, phosphocellulose and membrane-bound polypeptide analysis (b) are represented by means of sample rarefaction. S.d. bars are represented. Logarithmic tendency of polypeptide accumulation over 1000 hypothetical conditions following the one-band abundant protein (c) or in-depth analysis (d) strategies are shown. The top line in the (d) graph shows the limit corresponding to the total 4252 theoretical proteome of $R$. pomeroyi. (e) Powered tendency of new polypeptide detection in novel in-depth analysis (blue, left axis) and the false-positive percentage increase and extrapolated tendency when considering additional conditions (red, right axis).

covered over $48 \%$ of the theoretical chromosomally encoded proteome and $32 \%$ of the theoretical pDSS-3 megaplasmid-encoded proteome.

In essence, a complete functional genomic analysis of $R$. pomeroyi was possible using the extensive proteomic data compiled in this report. By comparing the proteomes detected for each of the conditions, we have defined essential proteins to face specific stresses. This includes not only the proteins already expected (for example, cold-shock proteins at low temperatures or chaperonin-like proteins at high temperatures), but also proteins with unknown functions that may have specific roles under given conditions, for example, SPO2567 in the presence of naphthalene and SPO1254 with port microbial communities. Some of these detected proteins could be used as bioindicators for contamination, such as benzoate determinants or copper resistance proteins detected in the port seawaters. Data obtained from the whole analysis also showed general variations due to different cellular physiological states or mechanisms to face common stresses (for example, ribosomal proteins or the proteins encoded on the pDDS3 megaplasmid in Figure 4). We found that $R$. pomeroyi's genome encodes for (i) proteins dedicated to specific conditions and (ii) others predicted for general physiological states and core proteome functions of the bacterium. In order to estimate the ratio of each group, we listed the proteins that were detected under all the conditions tested, those detected throughout the study and those never detected (Figure 5a). In the first place we found that around 600 proteins are always detected among the different in-depth conditions tested ( $\sim 15 \%$ of the theoretical proteome). This so-called core MS-detected proteome includes most of the house-keeping genes needed for marine Roseobacters (Supplementary Table S5, Moran et al., 2007; Tang et al., 2010). However, most free-living marine bacteria require a larger pool of functions to face their environment. We have shown that the number of proteins produced by $R$. pomeroyi to face a given environmental condition is around 1200-1400 polypeptides (each of the five in-depth analyses, Supplementary Table S4 $35 \%$ of its theoretical proteome)). It is noteworthy that this number of proteins would be very close to the whole proteome of the minimal oligotrophic marine bacterium C. pelagibacter ubique (Giovannoni et al., 2005). This bacterium is highly adapted to grow under very 
specific conditions found in the oligotrophic ocean. When we added the MS/MS data obtained from all the different physiological conditions tested, we compiled 1963 proteins ( $\sim 50 \%$ of the theoretical proteome of $R$. pomeroyi). Interestingly, Wegener et al obtained the same value (1955 polypeptides) when analysing the proteome of the freshwater cyanobacterium Synechocystis sp. PCC 6803 under 12 different environmental conditions (Wegener et al., 2010). This indicates that $30-35 \%$ of the theoretical proteome of $R$. pomeroyi when not including the 600 core proteome from the 1963 total detected) might be dedicated to to adaptation to general physiological changes (that is, exponentialstationary transition), environmental changes (that is, rich-poor media) or common stress response factors. Thus, we suggest that the remaining $50 \%$ of the theoretical proteome of $R$. pomeroyi seems to be dedicated to very specific functions and will be detected only if specific growth conditions are attempted (Figures $6 \mathrm{~b}$ and d).

The analysis carried out here has allowed us to define the genomic fractions of $R$. pomeroyi. Although the oligotrophic waters of the vast oceans are mainly dominated by oligotrophic-like organisms (Biers et al., 2009), in surface and coastal waters a higher predominance of bacteria with a generalist lifestyle is found (Mou et al., 2008; Lauro et al., 2009). The use of high throughput proteomics to analyse the detectable proteome of $R$. pomeroyi has revealed the basic need for a marine generalist in terms of proteome. Our data suggest that $\sim 30 \%$ of its theoretically encoded proteome is dedicated to what could be considered as the bare minimum proteome for living in a virtual environment without stress, energy and specific metabolite requirements, that is, what is generally considered as housekeeping functions. Whether the equivalent bare minimum proteome could be the same in specialist organisms remains to be established. Most but not all of these proteins are indeed orthologs and conserved among microorganisms. An additional $\sim 20 \%$ of the $R$. pomeroyi proteome is dedicated to adaptation to general cellular physiological variations and main specific physiological traits. In essence, this pool is also probably required in specialist organisms but could be much diverse in nature and proportion. This fraction could also be drastically reduced or modified in species such as C. pelagibacter ubique, where transitions from exponential to stationary phase have shown very small protein variations (Sowell et al., 2008). The remaining $50 \%$ of $R$. pomeroyi theoretically encoded proteome would be plausibly required to face very specific environmental changes as shown here. This 50\%-opportunitroph gene pool is possibly lacking or extremely reduced in specialist-like bacteria. Part of the $50 \%$-opportunitroph fraction could even just represent a gene pool in generalists for further long-term mutation-based adaptation of the population.
Our proteomic study shows that covering the full theoretical proteome of such generalist microorganisms is today a difficult challenge. Hundreds of different growth and stress conditions are probably necessary to detect the whole theoretical proteome of $R$. pomeroyi. This marine bacterium comprises a large toolbox to take advantage of a large number of lithoheterotrophic energy sources, interact with its environmental and cope with the potential toxic effects associated to different life conditions. We have shown here that half of its large genetic pool is probably used in very specific situations. Nevertheless, the products encoded by this fraction give enough adaptive advantage in the marine environment to be maintained in the genome.

\section{Acknowledgements}

JA Christie-Oleza was supported by a fellowship from the Fundación Ramón Areces. We thank the Commissariat à l'Energie Atomique et aux Energies Alternatives, the Fundación Ramón Areces and the Ministère des Affaires Etrangères et Européennes, République Française (Partenariat Hubert Curien, Picasso Program) for financial support. We also thank C Bruley (CEA-Grenoble) for providing the IRMa parser, Jean-Charles Gaillard and Olivier Pible (both from CEA-Marcoule) for help with data treatment. B Nogales and R Bosch acknowledge the financial support of the Spanish MICINN through project CTM2008-02574/MAR (with FEDER co-funding) and the Acciones Integradas program (FR2009-0106).

\section{References}

Baudet M, Ortet P, Gaillard JC, Fernandez B, Guerin P, Enjalbal C et al. (2010). Proteomics-based refinement of Deinococcus deserti genome annotation reveals an unwonted use of non-canonical translation initiation codons. Mol Cell Proteomics 9: 415-426.

Biers EJ, Sun S, Howard EC. (2009). Prokaryotic genomes and diversity in surface ocean waters: interrogating the global ocean sampling metagenome. Appl Environ Microbiol 75: 2221-2229.

Buchan A, Gonzalez JM, Moran MA. (2005). Overview of the marine roseobacter lineage. Appl Environ Microbiol 71: 5665-5677.

Burgmann H, Howard EC, Ye W, Sun F, Sun S, Napierala S et al. (2007). Transcriptional response of Silicibacter pomeroyi DSS-3 to dimethylsulfoniopropionate (DMSP). Environ Microbiol 9: 2742-2755.

Carvalho PC, Fischer JS, Chen EI, Yates JR, Barbosa VC. (2008). PatternLab for proteomics: a tool for differential shotgun proteomics. BMC Bioinformatics 9: 316 .

Christie-Oleza JA, Armengaud J. (2010). In-depth analysis of exoproteomes from marine bacteria by shotgun liquid chromatography-tandem mass spectrometry: the Ruegeria pomeroyi DSS-3 case-study. Mar Drugs 8: 2223-2239.

Clair G, Roussi S, Armengaud J, Duport C. (2010). Expanding the known repertoire of virulence factors produced by Bacillus cereus through early secretome profiling in three redox conditions. Mol Cell Proteomics 9: 1486-1498. 
de Groot A, Dulermo R, Ortet P, Blanchard L, Guerin P, Fernandez B et al. (2009). Alliance of proteomics and genomics to unravel the specificities of Sahara bacterium Deinococcus deserti. PLoS Genet 5: e1000434.

DeLong EF. (2009). The microbial ocean from genomes to biomes. Nature 459: 200-206.

Fuhrman JA. (2009). Microbial community structure and its functional implications. Nature 459: 193-199.

Giovannoni SJ, Tripp HJ, Givan S, Podar M, Vergin KL, Baptista D et al. (2005). Genome streamlining in a cosmopolitan oceanic bacterium. Science 309: 1242-1245.

Glass JI, Assad-Garcia N, Alperovich N, Yooseph S, Lewis MR, Maruf $M$ et al. (2006). Essential genes of a minimal bacterium. Proc Natl Acad Sci USA 103: $425-430$.

Gupta N, Benhamida J, Bhargava V, Goodman D, Kain E, Kerman I et al. (2008). Comparative proteogenomics: combining mass spectrometry and comparative genomics to analyze multiple genomes. Genome Res 18: 1133-1142.

Gustavsson N, Diez A, Nystrom T. (2002). The universal stress protein paralogues of Escherichia coli are coordinately regulated and co-operate in the defence against DNA damage. Mol Microbiol 43: 107-117.

Jaffe JD, Stange-Thomann N, Smith C, DeCaprio D, Fisher S, Butler J et al. (2004). The complete genome and proteome of Mycoplasma mobile. Genome Res 14: 1447-1461.

Kato JY, Funa N, Watanabe H, Ohnishi Y, Horinouchi S. (2007). Biosynthesis of gamma-butyrolactone autoregulators that switch on secondary metabolism and morphological development in Streptomyces. Proc Natl Acad Sci USA 104: 2378-2383.

Keller M, Hettich R. (2009). Environmental proteomics: a paradigm shift in characterizing microbial activities at the molecular level. Microbiol Mol Biol Rev 73: 62-70.

Koonin EV, Wolf YI. (2008). Genomics of bacteria and archaea: the emerging dynamic view of the prokaryotic world. Nucleic Acids Res 36: 6688-6719.

Lanfranconi MP, Bosch R, Nogales B. (2010). Short-term changes in the composition of active marine bacterial assemblages in response to diesel oil pollution. Microbial Biotechnol 3: 607-621.

Lauro FM, McDougald D, Thomas T, Williams TJ, Egan S, Rice S et al. (2009). The genomic basis of trophic strategy in marine bacteria. Proc Natl Acad Sci USA 106: 15527-15533.

Liu H, Sadygov RG, Yates 3rd JR. (2004). A model for random sampling and estimation of relative protein abundance in shotgun proteomics. Anal Chem 76: 4193-4201.

Moran MA, Buchan A, Gonzalez JM, Heidelberg JF, Whitman WB, Kiene RP et al. (2004). Genome sequence of Silicibacter pomeroyi reveals adaptations to the marine environment. Nature 432: 910-913.

Moran MA, Belas R, Schell MA, Gonzalez JM, Sun F, Sun $S$ et al. (2007). Ecological genomics of marine Roseobacters. Appl Environ Microbiol 73: 4559-4569.

Mou X, Sun S, Edwards RA, Hodson RE, Moran MA. (2008). Bacterial carbon processing by generalist species in the coastal ocean. Nature 451: 708-711.

Newton RJ, Griffin LE, Bowles KM, Meile C, Gifford S, Givens CE et al. (2010). Genome characteristics of a generalist marine bacterial lineage. ISME J 4: 784-798.
Paoletti AC, Parmely TJ, Tomomori-Sato C, Sato S, Zhu D, Conaway RC et al. (2006). Quantitative proteomic analysis of distinct mammalian Mediator complexes using normalized spectral abundance factors. Proc Natl Acad Sci USA 103: 18928-18933.

Polz MF, Hunt DE, Preheim SP, Weinreich DM. (2006). Patterns and mechanisms of genetic and phenotypic differentiation in marine microbes. Philosophical Transactions of the Royal Society B-Biological Sciences 361: 2009-2021.

Ramos JL, Gallegos MT, Marques S, Ramos-Gonzalez MI, Espinosa-Urgel M, Segura A. (2001). Responses of Gram-negative bacteria to certain environmental stressors. Curr Opin Microbiol 4: 166-171.

Scanlan DJ, Ostrowski M, Mazard S, Dufresne A, Garczarek L, Hess WR et al. (2009). Ecological genomics of marine picocyanobacteria. Microbiol Mol Biol Rev 73: 249-299.

Singer E, Webb EA, Nelson WC, Heidelberg JF, Ivanova N, Pati A et al. (2011). The genomic potential of Marinobacter aquaeolei-A biogeochemical opportunitroph. Appl Environ Microbiol 77: 2763-2771.

Sowell SM, Norbeck AD, Lipton MS, Nicora CD, Callister SJ, Smith RD et al. (2008). Proteomic analysis of stationary phase in the marine bacterium 'Candidatus Pelagibacter ubique'. Appl Environ Microbiol 74: 4091-4100.

Strom SL. (2008). Microbial ecology of ocean biogeochemistry: a community perspective. Science 320: 1043-1045.

Tang K, Huang H, Jiao N, Wu CH. (2010). Phylogenomic analysis of marine Roseobacters. PLoS One 5: e11604.

Ting L, Cowley MJ, Hoon SL, Guilhaus M, Raftery MJ, Cavicchioli R. (2009). Normalization and statistical analysis of quantitative proteomics data generated by metabolic labeling. Mol Cell Proteomics 8: 2227-2242.

VerBerkmoes NC, Denef VJ, Hettich RL, Banfield JF. (2009). Systems biology: Functional analysis of natural microbial consortia using community proteomics. Nat Rev Microbiol 7: 196-205.

Wagner-Dobler I, Biebl H. (2006). Environmental biology of the marine Roseobacter lineage. Annu Rev Microbiol 60: 255-280.

Wegener KM, Singh AK, Jacobs JM, Elvitigala TR, Welsh EA, Keren N et al. (2010). Global proteomics reveal an atypical strategy for carbon/nitrogen assimilation by a cyanobacterium under diverse environmental perturbations. Mol Cell Proteomics 9: 2678-2689.

Wilmes P, Bond PL. (2006). Metaproteomics: studying functional gene expression in microbial ecosystems. Trends Microbiol 14: 92-97.

Wilmes P, Bond PL. (2009). Microbial community proteomics: elucidating the catalysts and metabolic mechanisms that drive the Earth's biogeochemical cycles. Curr Opin Microbiol 12: 310-317.

York GM, Junker BH, Stubbe J, Sinskey AJ. (2001). Accumulation of the PhaP phasin of Ralstonia eutropha is dependent on production of polyhydroxybutyrate in cells. J Bacteriol 183: 4217-4226.

Zech H, Thole S, Schreiber K, Kalhofer D, Voget S, Brinkhoff $\mathrm{T}$ et al. (2009). Growth phase-dependent global protein and metabolite profiles of Phaeobacter gallaeciensis strain DSM 17395, a member of the marine Roseobacter-clade. Proteomics 9: 3677-3697. 